\title{
SYMMETRIC LINEAR MULTISTEP METHODS FOR SECOND-ORDER DIFFERENTIAL EQUATIONS WITH PERIODIC SOLUTIONS *
}

\author{
B.P. SOMMEIJER and P.J. VAN DER HOUWEN \\ Centre for Mathematics and Computer Science, Amsterdam, The Netherlands
}

B. NETA

Naval Postgraduate School, Monterey, CA, U.S.A.

\begin{abstract}
Special symmetric linear multistep methods for second-order differential equations without first derivatives are proposed. The methods can be tuned to a possibly a priori knowledge of the user on the location of the frequencies, that are dominant in the exact solution. On the basis of such extra information the truncation error can considerably be reduced in magnitude. Numerical results are compared with results produced by the symmetric methods of Lambert and Watson and the method of Gautschi.
\end{abstract}

Keywords: Numerical analysis, second-order differential equations, periodic solutions, linear multistep methods.

\section{INTRODUCTION}

In this paper we consider linear $k$-step methods

$$
\sum_{l=0}^{k} a_{l} y_{n+1-l}=h^{2} \sum_{l=0}^{k} b_{l} f\left(t_{n+1-l}, y_{n+1-l}\right), \quad k \geqslant 2, \quad n \geqslant k-1
$$

for integrating the initial value problem for the special second-order equation:

$$
\ddot{y}(t)=f(t, y(t)), \quad y\left(t_{0}\right)=y_{0}, \quad \dot{y}\left(t_{0}\right)=\dot{y}_{0} .
$$

This linear multistep $(L M)$ method is characterized by the polynomials

$$
\rho(\zeta):=\sum_{l=0}^{k} a_{l} \zeta^{k-l}, \quad \sigma(\zeta):=\sum_{l=0}^{k} b_{l} \zeta^{k-l} .
$$

Henceforth, we shall refer to (1.1) as the method $\{\rho, \sigma\}$.

The leading assumption of this paper is that it is a priori known that the solution is approximately of the form

$$
y(t) \approx C_{0}+\sum_{j=1}^{m} C_{j} e^{i \omega, t}
$$

with frequencies $\omega_{j}$ in a given interval $[\omega, \bar{\omega}]$.

The special case where $\omega_{j}=j \omega_{0}$, with $\omega_{0}$ given, was considered by GaUTSCHI [1]. His approach is essentially the following:

Let

* Report NM-R8501, Centre for Mathematics and Computer Science, Amsterdam. 


$$
\phi(z):=\rho\left(e^{z}\right)-z^{2} \sigma\left(e^{z}\right)
$$

then the local truncation error of (1.1) is given by [6]

$$
T_{n+k}=\phi\left(h \frac{d}{d t}\right) y\left(t_{n}\right) \text {. }
$$

Inserting (1.4) in (1.6) yields

$$
T_{n+k} \approx \phi(0) C_{0}+\sum_{j=1}^{m} C_{j} \phi\left(i h \omega_{j}\right) e^{i \omega_{j} t}, \quad \omega_{j}=j \omega_{0} .
$$

The coefficients in the polynomials $\rho$ and $\sigma$ are chosen in such a way that

$$
\phi\left(i h j \omega_{0}\right)=0, \quad j=0,1, \ldots, q
$$

for the largest possible value of $q$. Such methods are said to be of trigonometric order $q$ (see also [8]).

An alternative approach replaces the fitting conditions (1.8a) by the conditions

$$
\phi(0)=0, \quad \phi\left(i h \omega^{(j)}\right)=0, j=1,2, \ldots, q,
$$

where the $\omega^{(j)}$ are appropriately chosen points in the interval $[\omega, \bar{\omega}]$. For first-order differential equations this approach was analyzed in [4]. Following a similar anaTysis, we will try to minimize the function $|\phi(i h \omega)|$ in the interval $[\omega, \bar{\omega}]$ by a judicious choice of its zeros $i h \omega^{(j)}$ (see Section 2.2).

An advantage of the second approach (minimax-approach) over the Gautschi-approach is the increased accuracy in cases where no accurate estimate of $\omega_{0}$ is available or when the frequency is varying in time. In order to facilitate the use of these methods we also implemented a simple mechanism to estimate the frequency during the integration process. These aspects will be demonstrated by numerical experiments in Section 3.

In Section 2 we derive Gautschi- and minimax methods of optimal (algebraic) order of accuracy. As an example, for $k=4$ closed form expressions for the coefficients $a_{l}$ and $b_{l}$ of the polynomials $\rho$ and $\sigma$ are presented.

\section{SYMMETRIC METHODS}

A linear $k$-step method is called optimal if it is zero-stable and if its order equals $k+2$ [6]. It is well'nown [3] that optimal methods necessarily

i) are implicit,

(ii) have an even step number $k$,

(iii) have a symmetric $\sigma$-polynomial, i.e. $\sigma(\zeta)=\zeta^{k} \sigma\left(\zeta^{-1}\right)$

(iv) and have a $\rho$-polynomial the roots of which are 1 in modulus.

For second-order ODEs of the form (1.2) the last property implies that $\rho$ is symmetric so that from now on we will restrict our attention to symmetric, implicit $L M$ methods $\{\rho, \sigma\}$ with even step number $k$ (and consequently even order $p$ ). In passing we note that the implication $\rho$ is symmetric does not apply in case of first-order ODEs.

In his original paper, Gautschi considered Störmer-Cowell type methods, i.e. $\rho(\zeta) \rightarrow \zeta^{k}-2 \zeta^{k-1}+\zeta^{k-2}$ as $h \rightarrow 0$ which are clearly not symmetric for $k \geqslant 3$ and consequently have not the optimal (algebraic) order. Here, we propose $L M$ methods based on the Gautschi-approach as well as on the minimax-approach which do have the optimal order.

Our starting point is to choose a symmetric $\rho$-polynomial, which merely serves to let the method $\{\rho, \sigma\}$ be zero-stable. We require this polynomial to satisfy $a_{0}=1$ and $\rho(1)=\rho^{\prime}(1)=0$; hence it takes the form

$$
\rho(\zeta)=(\zeta-1)^{2} \prod_{l=1}^{(k-2) / 2}\left(\zeta-e^{i \theta_{l}}\right)\left(\zeta-e^{-i \theta_{l}}\right), \quad 0<\theta_{l}<2 \pi .
$$

Here, the $\theta_{l}$ are in principle free parameters; however, the condition of zero-stability restricts the multiplicity of $\theta_{l}$ to 2 if $\theta_{l} \neq \pi$ and to 1 if $\theta_{l}=\pi$. These free parameters can be used, for example, to 
decrease the error constant or to increase the interval of periodicity ([7]; see also Section 3, second example).

The $\sigma$-polynomial of a symmetric, implicit (even) $k$-step method has $\frac{1}{2} k+1$ free $b$-coefficients. To achieve order $p=k+2$, we have to satisfy $k+4$ conditions two of which are already fulfilled by the assumption $\rho(1)=\rho^{\prime}(1)=0$. Because of the symmetry of the method there remain $\frac{1}{2} k+1$ conditions to be met, equal to the number of free parameters. In constructing $L M$ methods based on the Gautschi-approach (as well as on the minimax-approach) we follow the same strategy but now the $b$ coefficients are determined by the fitting conditions (1.8a) (and (1.8b)). Because $\rho(1)=0$ implies $\phi(0)=0$, the number $q$ in (1.8) can be chosen equal to the number of free $b$-coefficients, i.e. $q=\frac{1}{2} k+1$.

The methods constructed in this way, converge for $h \rightarrow 0$ to the classical optimal method of order $2 q(=k+2)$. This is probably best understood using the following heuristic explanation: a classical optimal $k$-step $L M$ method can be considered as a method which is $(k+4)$ times fitted at the origin, or equivalently, $\phi(z)$ has a zero of multiplicity $k+4$ at $z=0$. On the other hand, the optimal Gautschi method has a $\phi$ - function with a double zero at $z=0\left(\rho(1)=\rho^{\prime}(1)=0\right)$ and $q=\frac{1}{2} k+1$ zeros at $z=i h j \omega_{0}$. However, because $\phi(i v)=0$ implies $\phi(-i \nu)=0$ the $\phi$ - functions of both types of methods have the same number of zeros. Finally, because the fitting points monotonically tend to zero for $h \rightarrow 0$, the fitting points will collapse in the limit, resulting in the same algebraic order. A similar argument holds for the minimax methods.

We remark that the procedure described above is equally valid in case of explicit $L M$ methods. If again symmetric methods are used, now a $k$-step method yields order $k$.

We conclude this section with a discussion of the solution of the linear system resulting from the fitting conditions (1.8a) and the minimax conditions (1.8b).

\subsection{Symmetric Gautschi methods}

Due to the symmetry of the method, $\phi(z)$ can be written as

$$
\phi(z)=\frac{1}{2} \sum_{l=0}^{k}\left(a_{l}-b_{l} z^{2}\right)\left(e^{(k-l) z}+e^{l z}\right)=e^{\frac{1}{2} k z} \sum_{l=0}^{k}\left(a_{l}-b_{l} z^{2}\right) \cosh \left(\left(\frac{k}{2}-l\right) z\right) .
$$

The fitting conditions (1.8a) assume the form

$$
\sum_{l=0}^{k}\left(a_{l}+b_{l}\left(j h \omega_{0}\right)^{2}\right) \cos \left(\left(\frac{k}{2}-l\right) j h \omega_{0}\right)=0, \quad j=1, \ldots, q,
$$

or, equivalently,

$\left(j h \omega_{0}\right)^{2}\left\{\sum_{l=0}^{\frac{k}{2}-1} 2 b_{l} \cos \left(\left(\frac{k}{2}-l\right) j h \omega_{0}\right)+b_{k / 2}\right\}=-\sum_{l=0}^{k} a_{l} \cos \left(\left(\frac{k}{2}-l\right) j h \omega_{0}\right), j=1, \ldots, q$.

It should be noted that only real-valued equations occur in this system. One possible way to solve this system is, of course, a numerical approach. However, if $h \omega_{0}$ is extremely small, this system is very badly conditioned and we will run into numerical difficulties. Therefore, it is convenient to have available closed form expressions for the solution of this system. To give an example, we derived for $k=4$ such expressions using REDUCE, a package used for performing symbolic as well as numerical mathematical manipulation [2]. The results are:

$$
b_{0}=-\frac{(x-1)}{36 \nu_{0}^{2}} \cdot \frac{2 x\left(16 x^{3}+38 x^{2}+24 x+3\right)+\alpha(5 x+4)}{x(x+1)(2 x+1)\left(4 x^{2}+2 x-1\right)}
$$




$$
\begin{aligned}
& b_{1}=-\frac{(x-1)}{9 \nu_{0}^{2}} \cdot \frac{2 x\left(20 x^{4}+60 x^{3}+40 x^{2}-3\right)-\alpha\left(18 x^{3}+14 x^{2}-3 x-2\right)}{x(2 x+1)\left(4 x^{2}+2 x-1\right)}, \\
& b_{2}=\frac{(x-1)}{18 \nu_{0}^{2}} \cdot \frac{2 x\left(40 x^{5}+12 x^{4}-56 x^{3}-20 x^{2}+6 x-3\right)+\alpha\left(108 x^{4}+170 x^{3}+42 x^{2}-25 x-4\right)}{x(x+1)\left(4 x^{2}+2 x-1\right)},
\end{aligned}
$$

where $x:=\cos \nu_{0}$ and $\nu_{0}:=\omega_{0} h$, and where $\alpha$ is the free parameter occurring in the $\rho$-polynomial

$$
\rho(\zeta)=(\zeta-1)^{2}\left(\zeta^{2}-\alpha \zeta+1\right)
$$

Evidently, zero-stability requires $-2 \leqslant \alpha<2$.

\subsection{Symmetric minimax methods}

Proceeding as in the previous section, the system defining the minimax methods reads (cf. (1.8b) and (2.4))

$\left(h \omega^{(j)}\right)^{2}\left\{\sum_{l=0}^{\frac{k}{2}-1} 2 b_{l} \cos \left(\left(\frac{k}{2}-l\right) h \omega^{(j)}\right)+b_{k} / 2\right\}=-\sum_{l=0}^{k} a_{l} \cos \left(\left(\frac{k}{2}-l\right) h \omega^{(j)}\right), \quad j=1, \ldots, q$,

where the $i \omega^{(j)} h$ are zeros of the function $\phi(i h \omega)$ such that it has a small maximum norm in the interval $\omega \leqslant \omega \leqslant \bar{\omega}$. First, we observe that $\phi(i h \omega)=\exp (i k h \omega / 2) \psi(i h \omega)$ with $\psi(i h \omega)$ real-valued. If $\psi(i h \omega)$ wouTd be a polynomial in $\omega$, then the optimal choice would be a shifted Chebyshev polynomial (cf. [4]). This suggests to approximate $\psi$ by such a Chebyshev polynomial and to identify the $\omega^{(j)}$ with the zeros of this polynomial. Since $\psi(i h \omega)$ is an even function of $\omega$ we write (cf. (2.2))

$$
\phi(i h \omega)=e^{\frac{i k h \omega}{2}} \psi(i h \omega) \approx e^{\frac{i k h \omega}{2}} C T_{q}\left(\frac{2 \omega^{2}-\bar{\omega}^{2}-\underline{\omega}^{2}}{\bar{\omega}^{2}-\underline{\omega}^{2}}\right),
$$

where $C$ is some constant. Thus

$$
\omega^{(j)}=\left[\frac{1}{2}\left(\bar{\omega}^{2}+\underline{\omega}^{2}\right)+\frac{1}{2}\left(\bar{\omega}^{2}-\underline{\omega}^{2}\right) \cos \left(\frac{2 j-1}{2 q} \pi\right)\right]^{\frac{1}{2}}, j=1, \ldots, q .
$$

\section{Numerical Illustration}

In this section the optimal Gautschi methods and the optimal minimax methods are tested for $k=4$ (resulting in (algebraic) order 6). They will be compared mutually and also with the 6th-order, 4-step (implicit) method of LAMBERT AND WATSON [7], which is also intended to integrate efficiently periodic initial value problems. Actually, this method is the conventional optimal 4-step method. Similar to the 'fitted' methods, it has one free parameter $\alpha$ (cf. 2.5). In all methods this parameter $\alpha$ is chosen equal to zero.

Additionally, we list the results of the original 4-step Gautschi method of trigonometric order 3 (cf. $[1$, p. 393]). This method has only algebraic order 5 because it lacks symmetry.

\subsection{Implementational details}

It will be clear that a proper application of the Gautschi- and minimax methods requires a more or less accurate estimate of the frequency $\omega_{0}$ and the frequency-interval $[\omega, \bar{\omega}]$, respectively. If these estimates are not available in advance, and also in cases where the frequency is varying in time, it will be convenient to have a mechanism to estimate the frequency automatically. Therefore, we implemented such a mechanism based on the following strategy: Suppose that the (local) solution on $\left[t_{n-1}, t_{n}\right]$ can sufficiently accurate be approximated by

$$
y(t) \simeq C_{0}+C_{1} e^{i \omega(n) t} \quad, \quad t \in\left[t_{n-1}, t_{n}\right] .
$$

Then, requiring $y\left(t_{i}\right)=y_{i}$ and $y^{\prime \prime}\left(t_{i}\right)=f_{i}, \quad i=n-1, n$, we obtain 


$$
(\omega(n))^{2}=\frac{f_{n-1}-f_{n}}{y_{n}-y_{n-1}} .
$$

Now, we use a 'fitting' method to perform the step from $t_{n}$ to $t_{n+1}$ if the following conditions hold true:

(i)

$$
(\omega(j))^{2}>(\epsilon / h)^{2}, \epsilon=0.02, j=n, n-1, n-2 .
$$

Only three $\omega$-values are involved because we focused on four-step methods. Evidently, $(\omega(j))^{2}$ should be positive to obtain a real value for the frequency; the reason to impose the more restrictive condition (i) is motivated by the fact that the 'fitted' methods converge to the LambertWatson method for $h \rightarrow 0$ and do not show a substantial gain for very small values of $h \omega(j)$. Moreover, the (numerical) solution of (1.8) gives numerical difficulties for $h \omega(j)<<1$

(ii)

$$
\max _{j}(\omega(j))<1.2 \min _{j}(\omega(j)), \quad j=n, \quad n-1, n-2 .
$$

The expression for the local truncation error $T_{n+k}$ (cf. Section 1) is based on constant $\omega$-values in the 'domain of influence' of the $L M$ method, i.e. $\left[t_{n-3}, t_{n+1}\right]$. To avoid a rigorous violation of this assumption, condition (ii) is applied.

If (i) and (ii) are satisfied we use $\omega_{0}=\tilde{\omega}, \tilde{\omega}:=(\omega(n-2)+\omega(n-1)+\omega(n)) / 3$ in case of the Gautschi-approach and $[\omega, \bar{\omega}]=[.95 \tilde{\omega}, 1.05 \tilde{\omega}]$ in case of the minimax-approach. If (one of) these conditions are not satisfied $\bar{w}$ e use the Lambert-Watson method to integrate this particular step from $t_{n}$ to $t_{n+1}$. In our experiments, the above strategy is applied every step. Finally, in the numerical tests the implicit relations were solved iteratively by Newton's method with the stopping criterion: residue less than $10^{-12}$. To measure the obtained accuracy we define

$$
c d(\psi(t)):=-\log _{10}\left(l_{2} \text { norm of the error of } \psi(t) \text { at } t=\text { tend }\right) .
$$

This quantity represents the number of correct digits of $\psi(t)$.

\subsection{Test examples}

Bessel's equation

First, we consider the frequently used test problem, originally discussed by GAUTSCHI [1]

$$
\ddot{y}(t)+\left(100+\frac{1}{4 t^{2}}\right) y(t)=0, \quad 1 \leqslant t \leqslant 10,
$$

with the initial conditions according to the 'almost periodic' particular solution

$$
y(t)=\sqrt{t} J_{0}(10 t)
$$

where $J_{0}$ is the Bessel function of the first kind. Obviously, the frequency of $y(t)$ is approximately 10 and consequently the Gautschi methods were applied using $\omega_{0}=10$. For the minimax-approach we used the frequency interval $[\omega, \bar{\omega}]=[9.5,10.5]$. Additionally, we applied these methods using the technique for automatically estimating the frequency. Table 3.1 shows the results for several step sizes. Compared with the symmetric methods, the original Gautschi method is by far inferior. This is not only due to its lower algebraic order but also a consequence of the fact that the coefficients are given in series form which is not accurate enough unless $h \omega_{0}<<1$. Moreover, this table clearly shows that it may pay to adapt the method to the knowledge available on the solution: for the versions in which the fitting points are fixed beforehand, the optimal Gautschi method has a global error which is approximately 100 times smaller than the error of the Lambert-Watson method, whereas the minimax method has a further increase in accuracy of about three decimal digits. Furthermore, we see that the 
TABLE $3.1 c d(y(t))$-values at $t e n d=10$ for problem (3.5), (3.6)

\begin{tabular}{cccccc}
\hline method & $\mathrm{k} / \mathrm{p} / \mathrm{q}$ & frequency & $h=1 / 10$ & $h=1 / 25$ & $h=1 / 50$ \\
\hline Lambert-Watson & $4 / 6 /-$ & - & 1.5 & 4.1 & 6.0 \\
optimal Gautschi & $4 / 6 / 3$ & 10 & 3.5 & 6.4 & 8.2 \\
optimal Gautschi & $4 / 6 / 3$ & automatic & 3.3 & 7.2 & 7.9 \\
& & & & & \\
optimal minimax & $4 / 6 / 3$ & {$[9.5,10.5]$} & 6.4 & 9.1 & 11.0 \\
optimal minimax & $4 / 6 / 3$ & automatic & 7.2 & 9.0 & 11.0 \\
original Gautschi & $4 / 5 / 3$ & 10 & 0.4 & 3.1 & 4.9 \\
\hline
\end{tabular}

automatic versions yield results which are approximately equal to the results obtained with a fixed frequency (interval). We mention that - in these tests - it did not occur that the algorithm changed to the Lambert-Watson method. The original Gautschi method gives the same results for both versions because its poor performance is not caused by an inaccurate estimate of the frequency.

\section{Orbit equation}

The next example was studied by Stiffel \& BeTris [9]:

$$
\ddot{z}(t)+z(t)=.001 e^{i t}, \quad z(0)=1, \quad \dot{z}(0)=.9995 i, \quad z \in \mathbb{C}, \quad 0 \leqslant t \leqslant 40 \pi .
$$

Writing $z(t)=u(t)+i v(t), u, v \in \mathbb{R}$, the solution is given by

$$
u(t)=\cos t+.0005 t \sin t, \quad v(t)=\sin t-.0005 t \cos t .
$$

The results of the various methods, when applied to the equivalent real-valued system for $u$ and $v$, are given in Table 3.2. As the solution possesses a frequency

TABLE 3.2. $c d(z(t))$-values at tend $=40 \pi$ for problem (3.7),(3.8)

\begin{tabular}{ccccccc}
\hline method & $\mathrm{k} / \mathrm{p} / \mathrm{q}$ & frequency & $h=\pi / 4$ & $h=\pi / 6$ & $h=\pi / 9$ & $h=\pi / 12$ \\
\hline Lambert-Watson & $4 / 6 /-$ & - & 1.5 & 2.6 & 3.7 & 4.5 \\
& & & & & & \\
optimal Gautschi & $4 / 6 / 3$ & 1 & 3.0 & 4.2 & 5.3 & 6.1 \\
optimal Gautschi & $4 / 6 / 3$ & automatic & 4.4 & 5.5 & 6.5 & 7.3 \\
& & & & & & \\
optimal minimax & $4 / 6 / 3$ & {$[0.9,1.1]$} & 5.0 & 6.1 & 7.2 & 8.0 \\
optimal minimax & $4 / 6 / 3$ & automatic & 6.2 & 7.4 & 8.5 & 9.2 \\
original Gautschi & $4 / 5 / 3$ & 1 & 0.2 & 1.4 & 2.6 & 3.4 \\
\hline
\end{tabular}

close to 1 , we applied the Gautschi-type methods using $\omega_{0}=1$ and the minimax method was given the frequency interval $[0.9,1.1]$. Again, both types of methods were also tested using the 'frequencyestimator'. The solution $z(t)$ represents a slightly perturbed circular orbit in the complex plane. Its distance to the origin is given by

$$
\gamma(t)=\left[u^{2}(t)+v^{2}(t)\right]^{\frac{1}{2}}=\left[1+(.0005 t)^{2}\right]^{\frac{1}{2}},
$$

hence $z(t)$ spirals slowly outwards. Many numerical methods yield approximations which spiral inwards for all values of $h$; these methods were termed 'orbitally unstable' by Stiefel and Bettis. (This 
is because the principle roots of the characteristic equation are inside the unit circle for any small $h$; for a detailed discussion on these aspects we refer to [7].) Lambert and Watson constructed $L M$ methods possessing a periodicity property as long as the product of $h$ and the frequency $\omega$ remains within a certain interval and called this the interval of periodicity. To give an example, the LambertWatson method we are testing in this section has an interval of periodicity $(0,60 / 11)$. Therefore, the accuracy of $\gamma$ is probably more interesting than the accuracy of $z$. We found for all methods and for all step sizes used a numerical value of $\gamma$ larger than 1, which is, at least qualitatively, in accordance with its theoretical behaviour. The $c d$-values can be found in Table 3.3.

TABLE 3.3. $c d(\gamma(t))$-values at tend $=40 \pi$ for problem (3.7)-(3.9)

\begin{tabular}{ccccccc}
\hline method & $\mathrm{k} / \mathrm{p} / \mathrm{q}$ & frequency & $h=\pi / 4$ & $h=\pi / 6$ & $h=\pi / 9$ & $h=\pi / 12$ \\
\hline Lambert-Watson & $4 / 6 /-$ & - & 3.0 & 4.1 & 5.2 & 6.0 \\
& & & & & & \\
optimal Gautschi & $4 / 6 / 3$ & 1 & 4.2 & 5.4 & 6.5 & 7.3 \\
optimal Gautschi & $4 / 6 / 3$ & automatic & 4.4 & 5.6 & 6.6 & 7.3 \\
& & & & & & \\
optimal minimax & $4 / 6 / 3$ & {$[0.9,1.1]$} & 6.4 & 7.6 & 8.7 & 9.4 \\
optimal minimax & $4 / 6 / 3$ & automatic & 7.5 & 8.7 & 9.7 & 10.5 \\
original Gautschi & $4 / 5 / 3$ & 1 & 0.2 & 1.5 & 2.7 & 3.6 \\
\hline
\end{tabular}

The results of both tables give rise to conclusions similar to those of the first example. Again, the estimates of the frequency were accepted in all steps and the results obtained are even better than in the case where we used a fixed frequency.

\section{Nonlinear equation}

Our third problem is taken from JAIN, KAMBO and GoEL [5] and reads

$$
\begin{aligned}
& \ddot{z}(t)+\left(1+a+a b e^{-2 i t}\right) z(t)-a e^{-i t} z^{2}(t)=0, \quad 0 \leqslant t \leqslant 20 \pi, \\
& z(0)=1+b, \quad \dot{z}(0)=i(1-b), z \in \mathbb{C} .
\end{aligned}
$$

The theoretical solution, with $z(t)=u(t)+i v(t)$, is given by

$$
u(t)=(1+b) \cos t, \quad v(t)=(1-b) \sin t
$$

Following Jain et al. we selected the parameter values $a=0.1$ and $b=0.1$. The Gautschi- and minimax methods were applied in both versions; in the non-automatic version we employed $\omega_{0}=1$ and $[\omega, \bar{\omega}]=[0.9,1.1]$, respectively. As the solution (3.11) possesses only one, constant frequency $\omega \equiv 1$, the Gautschi method, using $\omega_{0}=1$, integrated exactly, whereas the automatic version gives results which are nearly exact (relative to the machine-precision). The minimax method did not exactly fit the frequency $\omega=1$ (cf. (2.7)) but could obtain a substantial gain in accuracy when compared with the Lambert-Watson method. Table 3.4 gives the results for several step lengths.

\section{Mathieu's equation}

The last test example is a Mathieu equation, also possessing a periodic solution:

$$
\ddot{y}(t)+(a-2 q \cos (2 t)) y(t)=0, \quad 0 \leqslant t \leqslant 20, \quad y(0)=1, \quad \dot{y}(0)=0 .
$$

We do not have available an exact solution; however, it is known that the solution is of the form

$$
y(t)=e^{i \lambda t} P(t)
$$

where $P(t)$ is periodic with frequency 2 and $\lambda$ is the 'characteristic exponent' which depends on $a$ and 
TABLE 3.4. $c d(z(t))$-values at tend $=20 \pi$ for problem (3.10), (3.11)

\begin{tabular}{|c|c|c|c|c|c|}
\hline method & $\mathrm{k} / \mathrm{p} / \mathrm{q}$ & frequency & $h=\pi / 6$ & $h=\pi / 12$ & $h=\pi / 24$ \\
\hline Lambert-Watson & $4 / 6 /-$ & - & 3.1 & 5.0 & 6.8 \\
\hline optimal Gautschi & $4 / 6 / 3$ & 1 & exact & exact & exact \\
\hline optimal Gautschi & $4 / 6 / 3$ & automatic & 11.5 & 11.1 & 11.0 \\
\hline optimal minimax & $4 / 6 / 3$ & {$[0.9,1.1]$} & 6.6 & 8.5 & 10.2 \\
\hline optimal minimax & $4 / 6 / 3$ & automatic & 7.9 & 9.7 & 10.9 \\
\hline original Gautschi & $4 / 5 / 3$ & 1 & 2.0 & 3.9 & 5.7 \\
\hline
\end{tabular}

$q$. By choosing $a=3.7$ and $q=2.0$ we achieve that $\lambda \simeq 2$. Hence, we tested the Gautschi method with $\omega_{0}=2$ and the minimax-approach used the frequency interval [1.9,2.1]. Both methods performed only slightly better than the Lambert-Watson method. This is explained as follows: in spite of the periodicity of the function $P(t)$, the solution $y(t)$ is not of the form (1.4), at least not with a small value of $m$. This became clear in using the automatic version: in the greater part of the time steps the estimator could not find positive values for $\omega^{2}(n)$ (see also the conditions (3.3)) and even if such values were found in three subsequent time steps they varied too rapidly to be useful.

TABLE 3.5. $c d(y(t))$-values at tend $=20$ for example (3.12)

\begin{tabular}{cccccc}
\hline method & $\mathrm{k} / \mathrm{p} / \mathrm{q}$ & frequency & $h=1 / 10$ & $h=1 / 20$ & $h=1 / 40$ \\
\hline Lambert-Watson & $4 / 6 /-$ & - & 3.6 & 5.4 & 7.2 \\
& & & & & 6.4 \\
optimal Gautschi & $4 / 6 / 3$ & 2 & 4.6 & 5.0 & 5.3 \\
optimal Gautschi & $4 / 6 / 3$ & automatic & 3.7 & & \\
optimal minimax & $4 / 6 / 3$ & {$[1.9,2.1]$} & 4.0 & 5.8 & 7.5 \\
optimal minimax & $4 / 6 / 3$ & automatic & 3.7 & 5.5 & 7.1 \\
original Gautschi & $4 / 5 / 3$ & 2 & 2.8 & 4.3 & 5.9 \\
\hline
\end{tabular}

Therefore, apart from its use in estimating the unknown frequency of the (local) solution, this example clearly shows, that this frequency-estimation technique is at the same time useful to detect whether or not $y(t)$ is of the required form (1.4). If this premiss on $y(t)$ is not fulfilled the fitting- and minimax methods are not feasible and a conventional method should be used. The results of all methods are collected in Table 3.5. The reference solution at tend $=20$ as well as the starting values for the 4-step methods were calculated with an automatic Runge-Kutta code from the NAG-library using a very small tolerance parameter to control the local error.

\section{REFERENCES}

[1] W. GAUTSCHI, Numerical integration of ordinary differential equations based on trigonometric polynomials, Numer. Math., Vol. 3, 1961, pp. 381-397.

[2] A.C. HEARN (ed.), REDUCE user's manual, Rand Corporation, Santa Monica, CA, 1983.

[3] P. HenRICI, Discrete variable methods in ordinary differential equations, John Wiley \& Sons, New York, 1962.

[4] P.J. VAN DER HOUWEN and B.P. SOMMEIJER, Linear multistep methods with reduced truncation 
error for periodic initial-value problems, IMA J. Num. Anal., Vol 4, 1984, pp. 479-489.

[5] R.K. JAIN, N.S. Kambo and R. Goel, High-order one-step P-stable methods for the numerical integration of periodic initial value problems, J. Comp. Appl. Math., Vol 9, 1983, pp. 361-367.

[6] J.D. LAMBerr, Computational methods in ordinary differential equations, John Wiley, London, 1973.

[7] J.D. LAMBERT and I.A. WATSON, Symmetric multistep methods for periodic initial value problems, JIMA, Vol 18, 1976, pp. 189-202.

[8] B. NeTA and C.H. FORD, Families of methods for ordinary differential equations bases on trigonometric polynomials, J. Comp. Appl. Math., Vol 10, 1984, pp. 33-38.

[9] E. Stiefel and D.G. Betris, Stabilization of Cowell's method, Numer. Math., Vol 13, 1969, pp. 154-175. 